\title{
Index of Case Law
}

\section{ICSID Case Law}

Abaclat I

background 54

threshold $45^{-46}$

Abaclat II

background 54

threshold 47

Alpha Projektholding

background 57

threshold 39

Amco Asia

background $32,57,60$

threshold 32

Azurix

background $\quad 63-64$

Blue Bank

background 61

threshold 40

Brandes $65 n 325$

Burlington

background $54-55,68,70$

threshold 41

Caratube

background $70-71,72$

threshold 41-42

Cemex

background 79

CIT 70

CMS 69

ConocoPhillips I

background 74

threshold 37

ConocoPhillips II

background 55

threshold 42

ConocoPhillips III

background $55^{-56}, 74-75$

threshold 42

ConocoPhillips IV

background $74-75$

threshold 38,50

ConocoPhillips v background $74-76$

threshold 38-39, $5^{0}$

ConocoPhillips VI

background $74,76-77$

threshold $48,5^{\circ}$

Duke Energy International 63

EDF

background 80-81

threshold 39

Electrabel

background 64

threshold 44-45

Enron 69, 117

Favianca I

background $\quad 77-78$

Favianca II

background 78

threshold 49,50

Favianca III

background 78-79

threshold 49,50

Generation Ukraine background 61

Getma

background 51,80

threshold $46-47,51$

Highbury

background 166

threshold 166

Holcim 80

Hrvatska 139n754

Ickale

background 67-68

threshold 42-43

Kilic 68

Lemire

background 62 
LG\&E $\quad 117$

\section{Nations Energy \\ background $\quad 5^{8-59}$ \\ threshold 45}
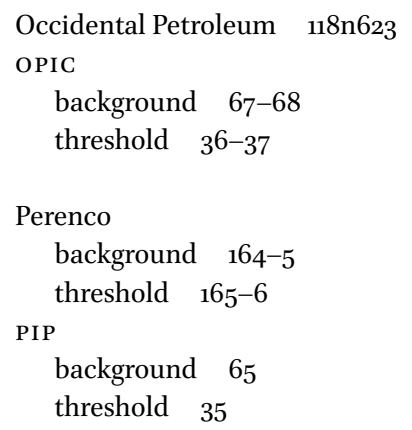

Repsol

background $59,68-70$

threshold 40

RFCC 119-120

Rusoro

background 72

Saba Fakes

background 72

Saint-Gobain

background $\quad 72,79$

threshold 47

Sempra 69

SGS

background 63

threshold 34

Siemens

Suez I

background $\quad 63-64$

background 71

threshold 43-44

Suez II

background $80-82$

threshold 35

S\&T Oil

background 80

Tanzania Electric background 73

Tidewater

background $\quad 65^{-67}$ threshold $\quad 36$

Total

background 59

threshold $\quad 47-48,5^{0-51}$

Transgabonais 65

Universal Compression

background $58,65^{-67}$

threshold 37

Urbaser

background 73

threshold $\quad 39-40$

Vannessa Ventures

background 60

Vivendi

background 33,60

threshold 33

Zhinvali

background 61

\section{UnCitral Case Law}

вG Group 117

background 117

Canfor 122

background 121

cc/Devas

background 119

threshold 119

EnCana 118

background 118

Eureko $123^{-124}$

background 123

Grand River $\quad 116$

background 115

ICS

background $\quad 116$

threshold $\quad 116$

National Grid $\quad 117$

background 117 
National Grid II

background 113-114

threshold 114

Ruby Roz $\quad 70-71$

Suez II (AWG)

background $80-82$

threshold 124
Telekom Malaysia

background 119

threshold 120-121

Vito Gallo

background 122

threshold 123 